\title{
Validating attentive locomotion training using interactive treadmill: an fNIRS study
}

\author{
Seunghue Oh, Minsu Song and Jonghyun Kim *D
}

\begin{abstract}
Background: Existing treadmill-based locomotion training, which has been used for gait function recovery, still has limitations, such as less attentive training. Interactive treadmills (ITMs) were developed to overcome these limitations, but it has not yet been verified that ITMs can make the user pay closer attention to walk training.

Methods: An experimental comparison between ITMs and conventional treadmills was conducted by measuring the level of the user's attention using functional near-infrared spectroscopy (fNIRS). To consider the effect of task complexity on the subject's attention, we provided two (slow and fast) speed conditions for walking on both treadmills.

Results: Both the cortical activity images and oxygenated hemoglobin (oxyHb) changes showed that the level of attention to walking induced by the ITM was significantly higher than that induced by the conventional treadmill. We found that the walking speed on the ITM also affected the level of attention.
\end{abstract}

Conclusion: ITM-based locomotion training would be a promising solution to the limitations of existing treadmill-based locomotion training currently used to improve gait function recovery.

Trial registration: DGIST-HR-150309-03-02. Registered 01 March 2015.

Keywords: Gait training, Attention, Task complexity, Walking speed, Interactive treadmill (ITM), fNIRS

\section{Background}

Recovery of gait function is a typical goal of rehabilitation $[1,2]$, and thus gait training for patients with impaired gait has been widely used in clinical practice [2-5]. Although the ultimate goal of gait training is to improve overground walking ability [6], gait training has mainly been conducted on treadmills $[5,7]$. This is because treadmills can safely and consistently provide task specific training in a limited space $[8,9]$, and treadmill walking is not significantly different from overground walking with respect to kinematic and kinetic aspects [10, 11]. However, treadmill-based locomotion training (TBLT) still has limitations on both physical and mental activity.

\section{Limitations on physical activity}

The kinematic and kinetic similarities between treadmill walking and overground walking were only verified with the treadmill belt turning at a constant speed $[10,11]$.

\footnotetext{
* Correspondence: jhkim@dgist.ac.kr

Department of Robotics Engineering, DGIST (Daegu Gyeongbuk Institute of

Science and Technology), 333 Techno Jungang-daero, Daegu 42988,

Republic of Korea
}

Since existing TBLT is mainly focused on walking at a constant speed $[1,5]$, walking on the treadmill can be regarded as similar to walking overground at a constant speed. However, it is clear that treadmill walking is a different physical activity compared with actual overground walking in which users decide their walking speed by themselves [8].

\section{Limitations on mental activity}

It is well-known that walking with a normal gait is a highly automated skill developed by natural practices during the growth period $[12,13]$. Hence, except for special circumstances (i.e. walking on ice), a healthy person can walk well using very little attention on walking $[13,14]$. However, for patients with impaired gait due to central nervous system injuries, it is difficult to maintain their normal gait, and thus it is necessary to re-establish their gait for locomotion [13]. Previous studies reported that a patient's mental activity during gait re-establishment, especially the external focusing of attention $[15,16]$, such as focusing on regulating gait speed, resulted in a better outcome for training [14-16]. In spite of the importance of the patient's 
attention being properly focused during rehabilitation, existing TBLT has been vulnerable to a lack of properly focused attention. TBLT, with a constant treadmill speed, provides a monotonous task for the user, who can easily become accustomed to the task, and would therefore be less attentive $[8,9]$.

There have been some attempts to overcome these limitations of existing TBLT. Several self-paced treadmill systems (i.e. TreadPort [17] and treadmill-on-demand [18]) were reported to enable the user to adjust the treadmill walking speed by using a treadmill belt speed controller based on the user's position measured either by an active mechanical tether $[17,19]$ or a non-contact sensor [18], or force sensors [20]. They commonly allow the user to accelerate or decelerate on the treadmill, and thus treadmill walking becomes more similar to overground walking in terms of physical activity. Along with the self-paced treadmill function for physical activity, they also provided external visual biofeedback, which would be beneficial to overcome the limitations of TBLT on mental activity $[17,19,20]$. However, these studies did not focus on the mental activity, so did not verify whether the attention, which is related to the mental activity, was really improved or not.

Recently, our research team has been developing an interactive treadmill (ITM) $[8,9,21]$ as an effective and efficient system when used with TBLT for gait rehabilitation. To overcome the limitation on physical activity cost-effectively, the self-paced treadmill function of the ITM was implemented based on a novel belt speed controller for minimizing the anomalous force generated by the user's acceleration or deceleration [8]. Moreover, thanks to the self-paced treadmill function, the ITM has provided an environment that causes the user to be more attentive during treadmill walking. Based on the assumption that proper biofeedback during locomotion training would improve the user's attention, this is achieved by including a protocol that causes the user to make efforts to achieve a target walking speed, which can be regarded as giving the user an external focus of attention [16], through visual biofeedback. Although providing an attentive environment is the main goal of the ITM, the effect of the ITM regarding the user's attention has not been verified yet, as the existing self-paced treadmill system did not [17, 19, 20]. It should be noted that there was an attempt to check the brain activation during treadmill walking on ITMs, but it did not investigate whether the attention was improved or not [22].

Based on the hypothesis that ITM-based locomotion training increases the user's attention to the training, the purpose of this study is to investigate whether this hypothesis can be confirmed or not. For that, we used functional near-infrared spectroscopy (fNIRS), a non-invasive method that indirectly monitors brain activation by measuring changes of hemoglobin $(\mathrm{Hb})$ in cerebral blood flow [23, 24], to observe what brain activation occurs in the cortical area related to gait. Compared with similar modalities to measure $\mathrm{Hb}$ changes, i.e. functional magnetic resonance imaging and positron emission tomography, the main advantages of fNIRS are 1) more ecological setting without strong immobility constraints $[25,26]$ and 2) less susceptible monitoring to motion artifacts as well as metallic materials [27]. Due to those advantages, fNIRS has been regarded as a promising neuroimaging technique for complex behavior with active motion [28-31], especially for locomotion/walking $[25,26,32]$. Hence, fNIRS has been widely used to observe brain activation in walking [25, 26, 32-38]. Moreover, fNIRS was widely applied to specific tasks regarding mental activity, such as cognitive-related tasks [31, 39-41] and dual task execution [31, 36, 37]. Based on the reports that the degree of mental activity, such as the level of attention due to task complexity, is closely related to the change of oxygenated $\mathrm{Hb}$ (oxyHb) $[29,39]$, fNIRS can also be used to measure the level of attention [36, 37, 41, 42]. Therefore, we have used fNIRS to investigate the user's attention to walking during ITM-based locomotion training, by measuring the change of oxyHb in several brain regions which are related to this type of attention.

\section{Methods}

\section{Interactive treadmill}

The ITM consists of a motion sensor (Kinect v1, Microsoft, USA), a treadmill (PPS MED, Woodway, USA), a treadmill belt speed controller, and a monitor to provide biofeedback (Fig. 1) [8, 9, 21]. In order to overcome the limitation of existing TBLT in terms of physical activity, the ITM enables the user to stay on the belt even when undergoing acceleration or deceleration. This is accomplished through the belt speed controller which uses feedback from the user's position, which is measured by the motion sensor. Moreover, the controller of the ITM, which can minimize the anomalous forces generated by the belt acceleration, allows the user to perform a more natural acceleration or deceleration, similar to overground walking [8].

In addition, based on the user's natural acceleration or deceleration on the ITM, we proposed the following gait training protocol to overcome the limitation of TBLT in terms of mental activity. The user can recognize his or her current walking speed and the target speed in real time by using the visual biofeedback displayed on the monitor (Fig. 2a), and they can attempt to match their speed to the target speed during the walking task. If the target speed is provided as shown in Fig. $2 \mathrm{~b}$, the user needs to do the following to achieve the target speed: 1 ) reach the speed through rapid acceleration or deceleration (period \#1 in Fig. 2b), and 2) maintain the speed 

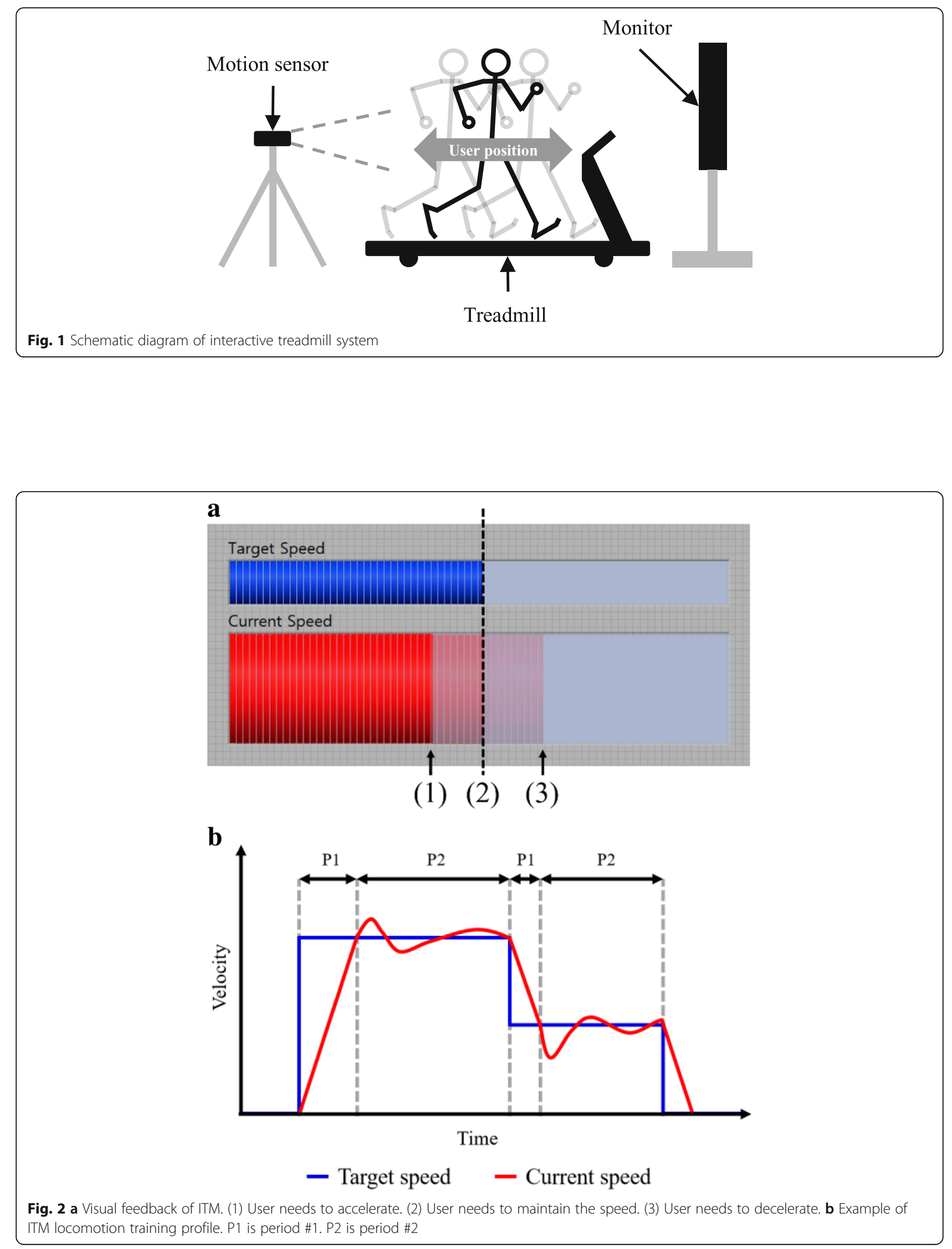
with gait adjustment (period \#2 in Fig. $2 b$ ). Note that the ITM requires the user's effort to maintain a constant walking speed. After all, the ITM was proposed as a simple and promising solution to overcome the limitations on mental activity during TBLT, because it provides a protocol to induce the user's continuous attention during TBLT, based on the characteristics of the ITM $[8,9,21]$.

\section{Experimental setup}

In this study, we compared the ITM with the conventional treadmill (CTM) to evaluate the performance of the ITM on inducing the user's attention. It is reported that the level of the attention is closely related to the task's complexity [39], and walking speed is a key factor of the task complexity when walking. Hence, we attempted to compare the levels of attention when using the ITM and the CTM with same walking speed. For that, with two target speeds (slow and fast), the comparison consisted of four tasks: ITM-slow, ITM-fast, CTM-slow, and CTM-fast.
Note that the target speeds of the CTM were the speed of the treadmill belt provided.

For each task, a block paradigm design that repeats three cycles, (with each cycle consisting of three phases, rest (20 s)-walking (60s)-rest (20 s)), was used to measure brain activation, as illustrated in Fig. 3. During the ITM-slow and ITM-fast tasks, we provided a target waking speed during the walking phase. During the cool-down mode, the ITM was activated to stop the ITM belt before the end of walking phase and the ITM displayed a zero-target speed (Fig. 3b). On the other hand, the belt speed profile which is to mimic the walking behavior of the ITM, as shown in Fig. 3c, was implemented for CTM-slow and CTM-fast tasks. The magnitude of the acceleration and deceleration of the profile was set to $0.3 \mathrm{~m} / \mathrm{s}^{2}$ (Fig. 3c).

The reason we conducted the experimental comparison with two different walking speeds (slow and fast) was to verify how the task complexity felt by the user affects the

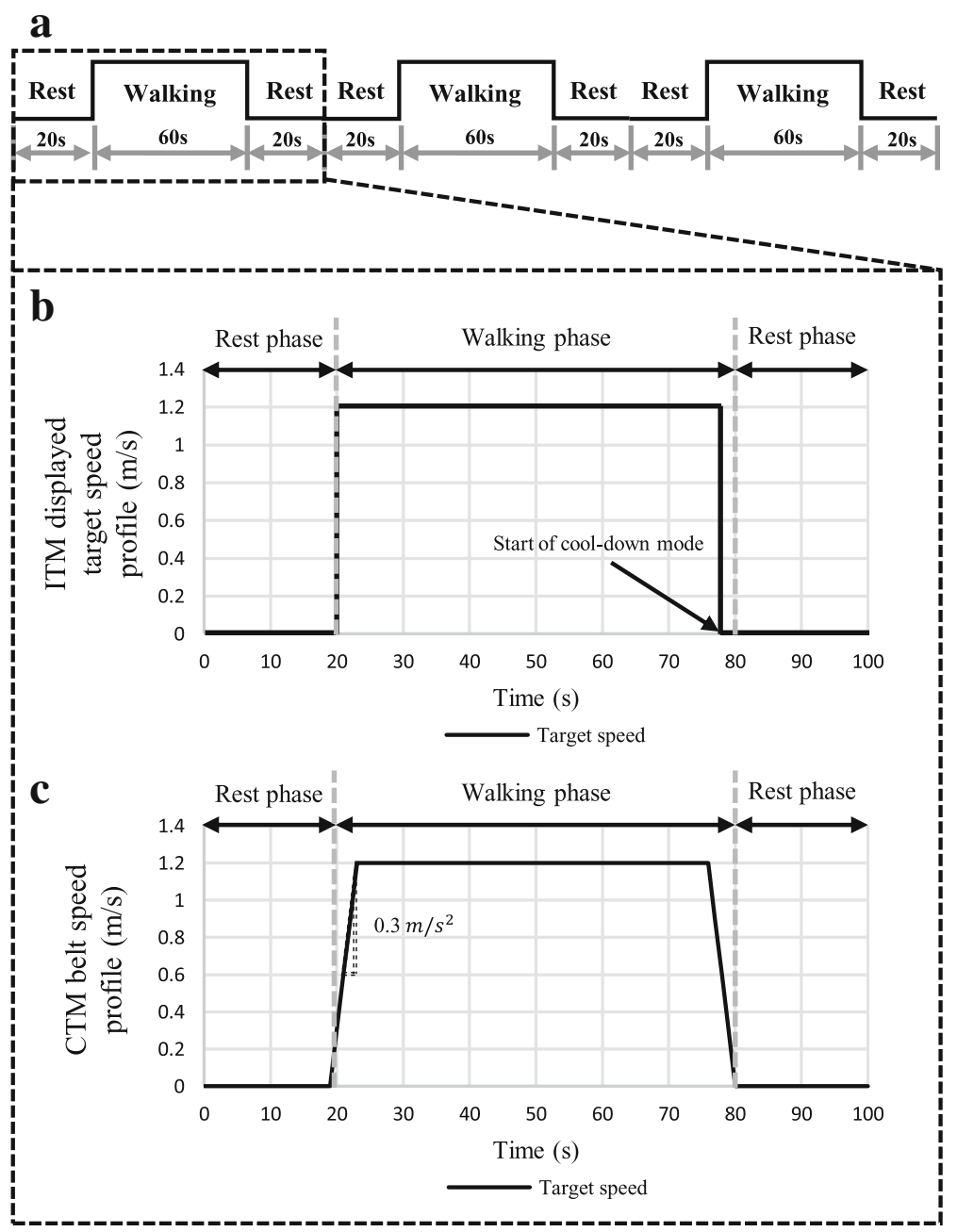

Fig. 3 a Block paradigm design. b Example of ITM displayed target speed profile. c Example of CTM belt speed profile 
user's level of attention induced by the ITM. For that, before the experiment, we obtained each subject's preferred walking speed by calculating his or her average speed during a $10 \mathrm{~m}$ overground walk, and the slow and the fast walking speeds for each subject were determined as follows: the fast speed was set to $125 \%$ of the preferred speed, and the slow speed was $75 \%$ of the preferred speed [8]. The difference in the user's level of attention when using the ITM or the CTM needed to be compared under similar work load intensity by considering the individual's gait capacity [43]. The preferred speeds are different for each subject, and thus the task complexity felt by the subject would not be same if fixed target speeds were provided to all subjects.

\section{Participants}

Twenty healthy subjects, who did not have any musculoskeletal or nervous system disease, were recruited. The participant demographics were summarized in Table 1. All participants were given the full instructions of the study and agreed to participate in the experiment. The experiment was conducted with the IRB (DGIST-HR-150309-03-02) approval of DGIST (Daegu Gyeongbuk Institute of Science and Technology). Figure 4 shows the subjects' preferred walking speeds as measured by a $10 \mathrm{~m}$ overground walk.

\section{Apparatus}

The ITM system used for this experiment is shown as Fig. 5. For the comparison between the ITM and the CTM, the treadmill of ITM system (without self-paced treadmill function) also used as CTM. Figure 3c shows the belt speed profile of CTM.

By using a fNIRS system (LABNIRS, Shimadzu, Japan) that has continuous-wave laser diodes with 780, 805 and $830 \mathrm{~nm}$ wavelength, the subject's cortical activation was observed at a sampling rate of $16 \mathrm{~Hz}$ [44]. We used a well-known international $10-20$ system to locate the fNIRS optodes [45]. The cranial vertex $(\mathrm{Cz})$ was set as a reference point for the optode placement. For accurate fixation, the optodes were mounted on the holder in the NIRS cap, and the distance between the pair of light source and detector optodes was set to $3 \mathrm{~cm}$. After acquisition of the fNIRS data, the coordinates of all the optodes and the positions of the anatomical landmarks

Table 1 Participant demographics

\begin{tabular}{ll}
\hline Characteristics & \\
\hline $\begin{array}{l}\text { Age (years) } \\
\text { Gender }\end{array}$ & male: $16 \pm 2.54$; range: $21-34$ \\
Physical activity level & $\begin{array}{l}\text { healthy (no underlying orthopedic } \\
\text { or neurological disorders) }\end{array}$ \\
Preferred speed (m/s) & $1.25 \pm 0.13$ \\
Education level (years) & $17.5 \pm 1.9$ \\
\hline
\end{tabular}

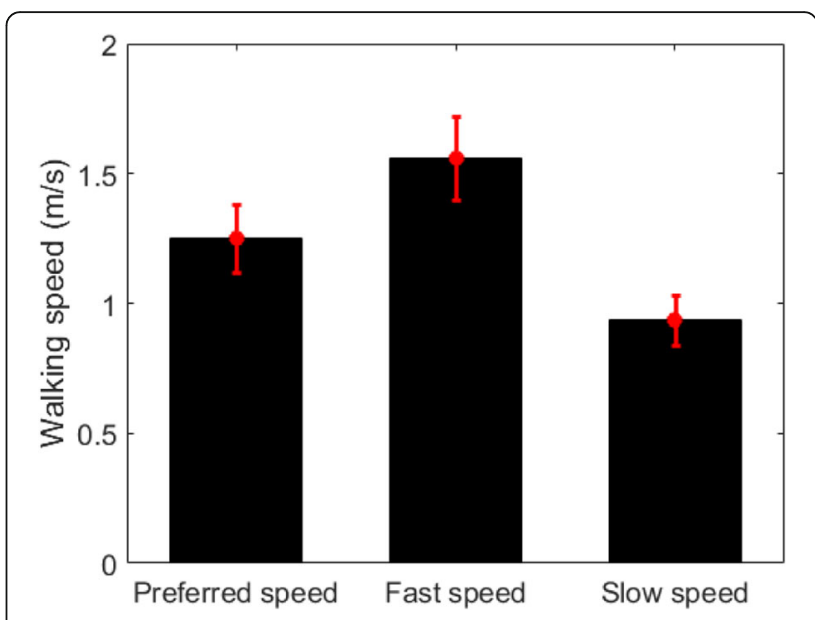

Fig. 4 Preferred walking speeds measured on a $10 \mathrm{~m}$ overground walk

(nasion, $\mathrm{Cz}$, left and right pre-auricular points) were obtained by using Fastrak System (TX-2, Polhemus, Colchester, VT, USA), based on the Montreal neurological institute standard brain space [46].

The purpose of this experiment is to observe the subject's level of attention to walking. It is well-known that the cognitive region of the prefrontal cortex (PFC) is related to general attention [36, 41, 47]. However, we cannot guarantee that by observing the PFC the attention measured is always the attention to walking because the subject could perform dual tasks while walking by mistake. Thus, the additional features, which are related to the attention, need to be monitored; task complexity felt by subject would determine the level of attention [39], and variability of gait parameters could possibility cause the subject to focus the attention which is measured [48]. Therefore, we chose the region of interest (ROI) based on the Brodmann area, as follows: cognitive region of the prefrontal cortex (PFC) [36, 41, 47], sensorimotor cortex (SMC) (which is related to task complexity) [39, 42], and supplementary area (SMA) and premotor cortex (PMC), which are related with variability of gait parameters $[43,48]$. In order to measure those regions, 20 nodes $(5 \times 4)$ with 31 channels were used, as illustrated in Fig. 6.

\section{Protocol}

After determining the subjects' target speeds based on his or her preferred walking speed measured prior to the experiment, we attached the fNIRS optodes to the subject who was sitting comfortably in a chair and wearing a NIRS cap on his or her head. After that, the subjects were asked to try about three minutes of walking on the ITM and the CTM to become accustomed to those experimental environments. The subjects conducted four tasks (ITM-slow, ITM-fast, CTM-slow, and CTM-fast) in randomized order. Each task was performed with the block paradigm design (Fig. 3a), as follows. When each task started, the subject stood on the treadmill for $20 \mathrm{~s}$ 


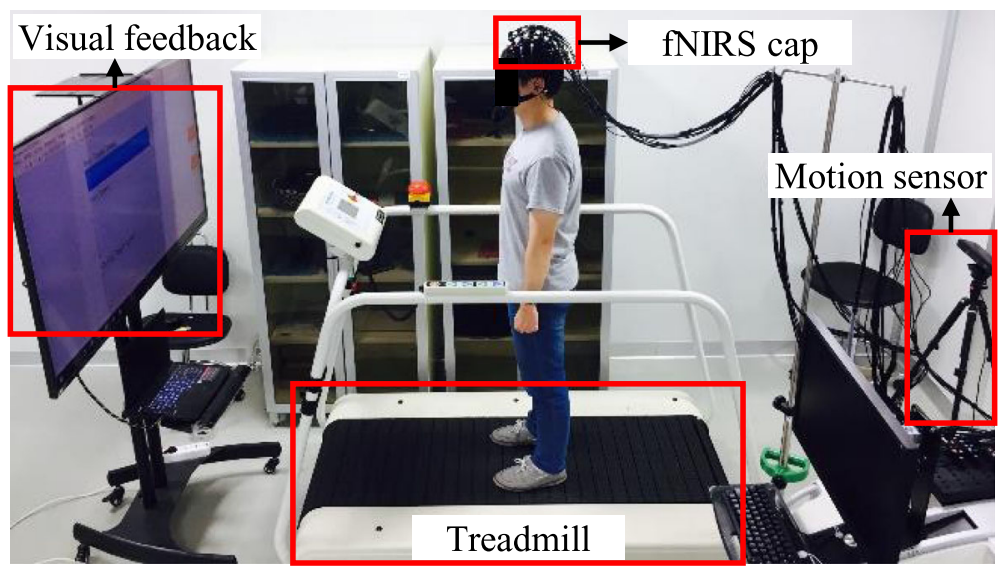

Fig. 5 Experimental setup

as a rest phase (rest in Fig. 3a), then walked for $60 \mathrm{~s}$ after we gave the verbal instruction, 'start' (walking in Fig. 3a). During the walk on the ITM, the subjects were instructed to match their walking speed to the target speed by using the visual biofeedback provided by the monitor which displayed the walking speed as well as the target speed in real-time (Fig. 2a). As to the walk on the CTM, the treadmill belt moved at the pre-defined speed profile (Fig. 3c) and there was no display on the monitor. Note that the subjects were allowed to shake their arms naturally during walking. After that, the subject waited for $20 \mathrm{~s}$ while standing on the treadmill until the next walking phase started (rest in Fig. 3a). That rest-walking-rest cycle was repeated three times for each task (Fig. 3a).

\section{Data analysis}

We have mainly used oxyHb for data analysis because oxyHb is known to be sensitive to task-related changes $[33,49]$. Based on the oxyHb measured by fNIRS, we

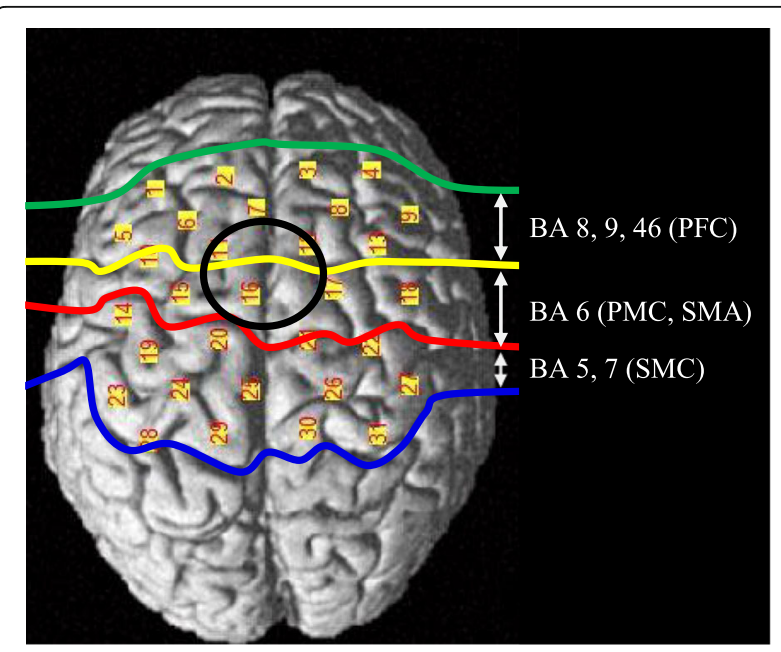

Fig. 6 NIRS channels and regions of interest (ROI) obtained optical imaging of cortical activities to show the location of the activation, and calculated the changes of oxyHb which can represent the degree of cortical activation quantitatively. NIRS_SPM, a MATLAB-based software package for statistical analysis of fNIRS, was used [46], and Gaussian smoothing with a full width at half maximum of $2 \mathrm{~s}$ was applied for noise compensation. In addition, Wavelet-MDL-based detrending was used to compensate for motion artifacts due to the subject's movement, such as breathing [50]. General linear model (GLM) analysis with a canonical hemodynamic response curve was performed to model the hypothesized $\mathrm{HbO}$ response under the experimental condition $[46,50]$.

For group analysis of the optical imaging of cortical activities, the interpolated activity map over the cortical surface from the $\mathrm{T}$ statistics calculated at discrete channels were computed by using NIRS-SPM, and for a stricter analysis, $\mathrm{HbO}$ was considered significant at $p<0.05$. The oxyHb changes for the regions of interest (PFC, SMC, SMA, and PMC), which were expressed in millimolar-millimeter units, and were obtained by summing the oxyHb changes of the channels included in the respective region. Here, the oxyHb change of each channel was calculated by subtracting the average oxyHb in the initial $10 \mathrm{~s}$ range of the rest interval from the average oxyHb in all task intervals. By using SPSS software (SPSS Inc. released 2006. SPSS for Windows, Version 15.0, SPSS Inc., Chicago, IL, USA), one-way ANOVA was applied to determine whether there was a statistically significant difference in the oxyHb change in each region of interest due to task differences $(p<0.05)$. In addition, we calculated the effective $\operatorname{size}(d)$ to evaluate the difference [51]. In general, the effect size $(d)$ is generally not important if $d<0.2$, small $\geq 0.2$, medium $\geq 0.5$, large $\geq 0.8$ and very important when it exceeds 1 [41]. 


\section{Results}

Optical imaging of cortical activities

The group analysis result of the optical imaging of cortical activities is shown in Fig. 7. Except for the CTM with fast speed, significant cortical activation was observed in all ROIs. Note that there was cortical activation of the SMC, SMA, and PMC regions when measuring the CTM with fast speed (Fig. 7). Regardless of walking (target) speed, cortical activation under the ITM occurred in a broader area than that in the CTM (Fig. 7). As to the PFC region related to the attention [36, 41, 47], the ITM always showed the activation while the CTM did not with fast speed, and with slow speed, the activation under the ITM covered a broader area of the PFC region than it did in CTM.

\section{Change of $\mathrm{OxyHb}$}

Figure 8 shows the comparison of OxyHb changes between the ITM and the CTM. First, in the fast speed, the oxyHb change in the ITM was larger than that in the CTM, and the differences of the oxyHb changes between the ITM and the CTM were significant in all regions $(p<$ 0.05 ) (PFC: $\mathrm{F}=2.70, d=1.38$; SMC: $\mathrm{F}=2.45, d=1.37$; SMA: $\mathrm{F}=2.28, d=1.08$; PMC: $\mathrm{F}=3.09, d=1.84$ ) (Fig. 8a). In the slow speed, the oxyHb change of the ITM was larger than that of the CTM in the PMC, SMA, and SMC regions, but the differences were not significant except in the SMA region ( $p<0.05 ; \mathrm{F}=2.21, d=1.24$ ) (Fig. $8 \mathrm{~b}$ ).

To investigate the effect of walking speed, we checked the oxyHb change in each treadmill environment according to target speeds (slow and fast speeds). The ITM showed larger oxyHb increases in fast speed than in slow speed, and the difference of the oxyHb increases was significant in the PFC, SMC and PMC regions $(p<0.05)$ (PFC: $\mathrm{F}=2.19$, $d=0.94 ; \mathrm{SMC}: \mathrm{F}=2.27, d=1.04 ; \mathrm{PMC}: \mathrm{F}=2.27, d=0.98$ ) (Fig. 9a). On the other hand, the CTM did not show any significant difference in all regions (Fig. 9b).

\section{Discussion}

From the experimental comparison, we found that both the optical imaging of cortical activities and change of oxy $\mathrm{Hb}$ are supporting our hypothesis; ITM walking increases the user's attention to walk, compared with CTM walking.

In the optical imaging of cortical activities shown in Fig. 7, one can see that the broader activation of all ROIs (PFC, SMC, SMA, and PMC) occurred under the ITM. Since the PFC and SMC regions are related to attention (control of attentional resources) [36, 41, 42, 47] and task complexity with voluntary movement [39], respectively, this broader activation of the PFC and SMC regions represents the subject's increased attention under the ITM walking. Of course, the increased attention measured could be different from attention to walking. However, the broader activation of the SMA and PMC regions, which are related to variability of gait parameters [48], can be regarded as indirect evidence that walking is the focus of the increased attention because the activation of the SMA and PMC regions would come from the effort to match the target speed by modulating the gait parameters. It is noteworthy that there was no PFC activation under the CTM with fast speed. This lack of activation under the CTM represents the current characteristics of existing TBLT.

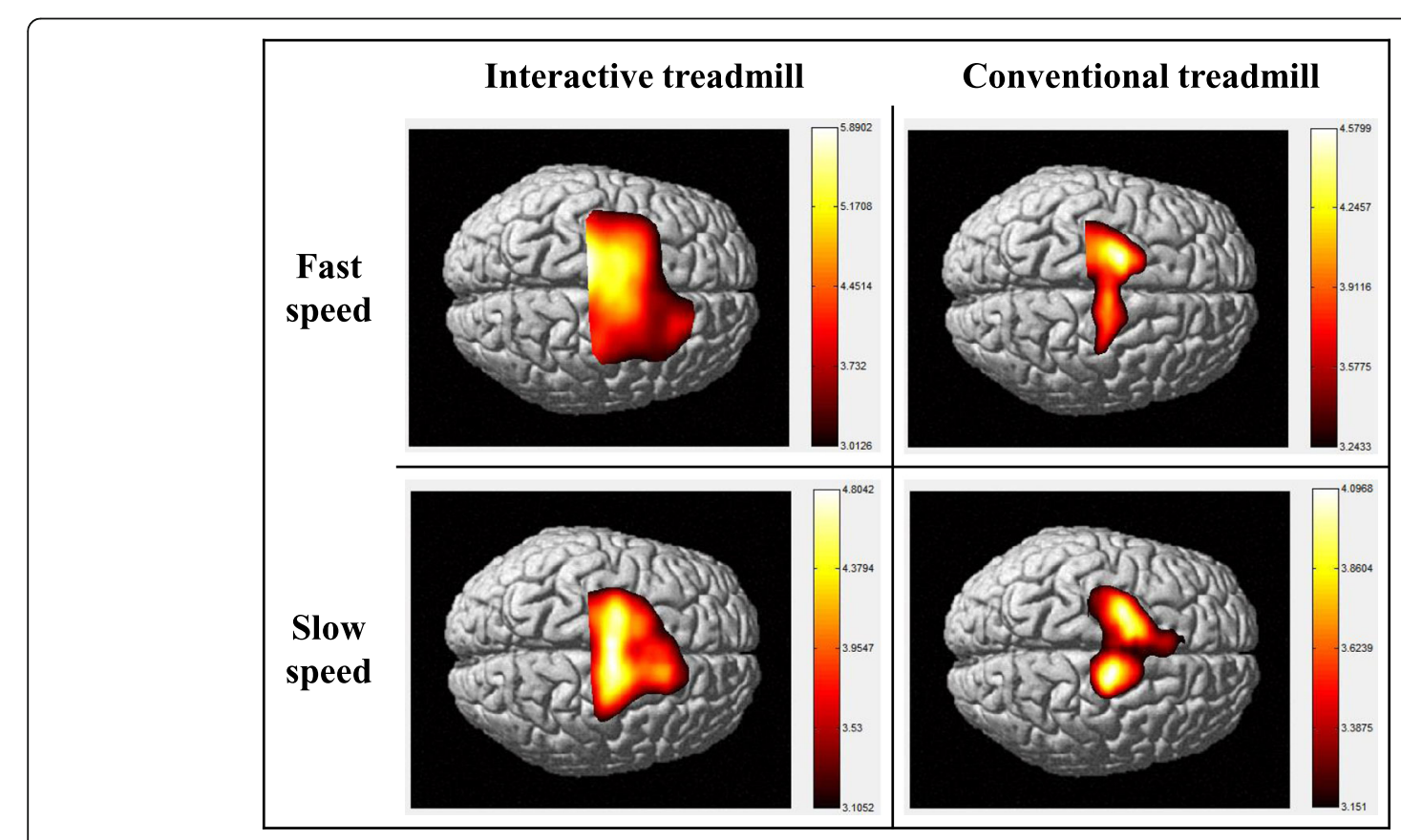

Fig. 7 Optical imaging of cortical activities (group analysis) 

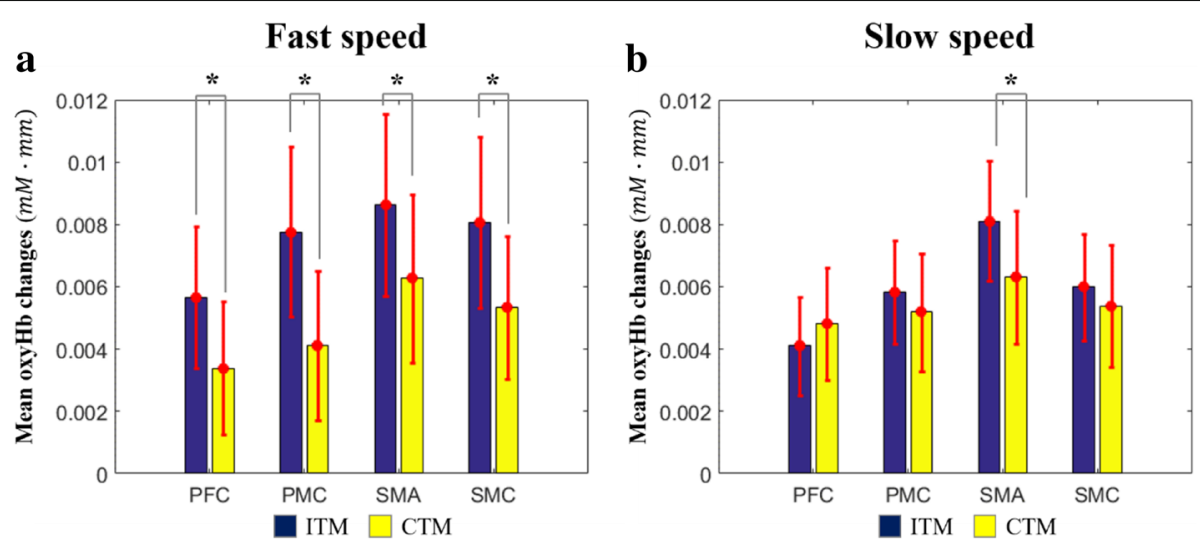

Fig. 8 Mean oxyHb changes. PFC: Prefrontal cortex, PMC: Premotor cortex, SMA: Supplementary motor cortex, SMC: Sensory motor cortex, oxyHb: oxy-hemoglobin, $\mathrm{mM} \cdot \mathrm{mm}$ : millimolar - millimeter

The results of the oxyHb changes also show that the ITM causes more attention to be used for walking than the CTM. The significant oxyHb increase of all ROIs under the fast speed clearly shows a larger level of attention under the ITM than that under the CTM (Fig. 8a). On the other hand, under slow speed, the significant increase occurred in the SMA region only (Fig. 8b). From the functions of the ROIs (i.e. SMC) [39], it would be because the subjects feel there was no significant difference of task complexity between the ITM and the CTM walking due to slow speed, while the ITM still needs more effort to maintain the target speed by modulating gait parameters than the CTM. This result implies that trying to make the user give more attention to walking (training) with the ITM-based locomotion training would be more effective with a fast target walking speed. It should be noted that the fast target speed is not absolute but relative, according to the user's preferred speed (or gait capacity).

The oxyHb increases of most ROIs under the fast target speeds (Fig. 9a) also supports the characteristics of the ITM above, the relationship between the attention to walking and the target walking speed. In contrast, the CTM did not show any difference according to walking speeds (Fig. 9b), which was already reported [35]. This result shows that the ITM can induce attentive training by providing appropriate target walking speed, which cannot be done with the CTM.

The dual task with walking could be more attentive than simple walking. There were several fNIRS studies to investigate the effect of the dual task, and their results showed larger brain activities than simple walking [32, 37, 52, 53]. Moreover, similar result was reported in a study with a different imaging modality, transcranial doppler (TCD) [54]. Note that TCD was applied to a cognitive-related task or dual task in walking in order to monitor brain activation by measuring cerebral blood flow $[54,55]$. Those results are consistent with our result that ITM walking, which would be more attentive than CTM walking, showed broader brain activation as well as significant oxyHb increase.
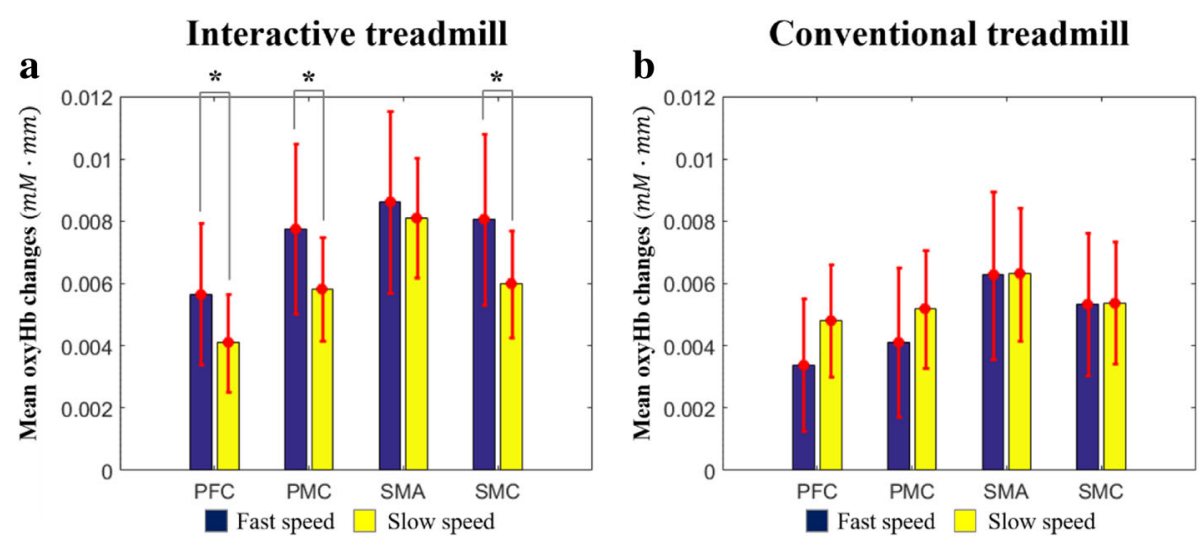

Fig. 9 Mean oxyHb changes. PFC: Prefrontal cortex, PMC: Premotor cortex, SMA: Supplementary motor cortex, SMC: Sensory motor cortex, oxyHb: oxy-hemoglobin, $m M \cdot m m$ : millimolar - millimeter 
There was an ANIRS study that observed increased PFC activity during CTM walking compared with overground walking in elderly [56], and this observation supports that overground walking would have less attentional resources than CTM walking. On the other hand, our study shows that ITM walking, which was to make it as similar as possible to overground walking in terms of physical activity, causes more attention than CTM walking in young healthy subjects. In spite of the different population of those studies, one can expect that the improved attention of ITM does not come from its physical function (self-paced treadmill) but from its training environment (protocol with appropriate biofeedback).

The proposed protocol of ITM-based locomotion training consists of acceleration, maintaining speed, and deceleration (Fig. 2b) [8, 9, 21]. Hence, we designed the walking phase of the block paradigm to contain those components (Fig. 3). Here, the maintaining speed period of the walking phase was much longer than acceleration or deceleration periods. This was done to mainly focus on investigating the effect of the ITM on the user's attention in the maintaining speed period. Even though the rapid acceleration and deceleration are very stimulating periods, the maintaining speed period of the ITM protocol is usually much longer than the acceleration or deceleration periods.

In the experiment, we conducted ITM and CTM walking under two conditions which included slow and fast walking speeds. Since the conditions were adopted to investigate the effect of the task complexity felt by the user, those speeds for each subject were determined based on his or her preferred walking speed. The variability of the preferred speeds displayed in Fig. 4 shows that it is appropriate to consider each subject's individual gait capacity, which is closely related to brain activation [43]. Using fixed speeds for all subjects could not achieve this.

From this study, it was verified that ITM-based locomotion training can result in more attentive gait training. This result shows that ITMs could be a promising solution to overcome the limitations of existing TBLT, especially regarding mental activity. This study still have some rooms to be improved. Although the expected target users of ITM-based locomotor training are elderly and patients with gait disorder, the hypothesis was validated with twenty young healthy subjects only. Moreover, due to the limitation of our fNIRS equipment, we did not use the short-separation channels, which is a promising method to correct the fNIRS signal $[32,57]$. In the future, similar verification of ITMs with the short-separation channels should be performed with elderly and the patients, especially with central nervous system injuries to consider different activation patterns due to their brain lesions [25]. In addition, to investigate the gait recovery caused by the ITM-based locomotion training, the change of gait performance with ITM training needs to be evaluated by using an ambulatory gait monitoring system [58].

\section{Conclusion}

By comparing the ITM walking with the CTM walking using fNIRS, we found that 1) the ITM causes significantly more attentive walking than the CTM and 2) the level of attention due to the ITM is affected by the target walking speed used. Along with kinematic/kinetic similarities, this attentive (non-automatic) training due to ITM-based locomotion training would result in better recovery of gait function for patients with central nervous system injuries.

\section{Abbreviations}

CTM: conventional treadmill; Cz: cranial vertex; fMRI: functional magnetic resonance imaging; fNIRS: functional near-infrared spectroscopy;

GLM: general linear model; $\mathrm{Hb}$ : hemoglobin; IRB: institutional review boards; ITM: interactive treadmill; oxyHb: oxygenated hemoglobin; PET: positron emission tomography; PFC: prefrontal cortex; PMC: premotor cortex; ROI: region of interest; SMA: supplementary area; SMC: sensorimotor cortex; TBLT: treadmill-based locomotion training

\section{Acknowledgments}

The authors would like to thank all subjects for the study.

\section{Funding}

This work was supported in part by the DGIST R\&D Program of the Ministry of Science, ICT, and Future Planning (18-BD-0401), and in part by the National Research Foundation of Korea (NRF) grant funded by the Korea government (MSIP) (No.2017R1C1B2010284).

Availability of data and materials

Data and materials can be made available upon request to the authors.

\section{Authors' contributions}

JK supervised the study. SO and JK conceptualized and designed the study. JK acquired the funding and provided the resources for the study. MS and JK implemented the interactive treadmill system. SO and MS designed the experiments. SO and MS prepared IRB for the experiments. SO acquired the data. SO and JK processed and analyzed the data from the experiments, and interpreted results from the data. SO drafted the original manuscript. JK finalized the manuscript. All authors read and revised the manuscript, and approved the final manuscript for publication.

Ethics approval and consent to participate

All subjects signed an informed consent approved by the Daegu Gyeongbuk Institute of Science and Technology IRB prior to the experiment (NO. DGISTHR-150309-03-02., registered 03. 01. 2015., https://larc.dgist.ac.kr/).

\section{Consent for publication}

Not applicable.

\section{Competing interests}

The authors declare that they have no competing interests.

\section{Publisher's Note}

Springer Nature remains neutral with regard to jurisdictional claims in published maps and institutional affiliations. 
Received: 17 July 2018 Accepted: 7 December 2018 Published online: 20 December 2018

\section{References}

1. Dobkin B, Barbeau H, Deforge D, Ditunno J, Elashoff R, Apple D, Basso M, Behrman A, Fugate L, Harkema S. The evolution of walking-related outcomes over the first 12 weeks of rehabilitation for incomplete traumatic spinal cord injury: the multicenter randomized spinal cord injury locomotor trial. Neurorehabil Neural Repair. 2007:21:25-35.

2. Barbeau $H$, Wainberg $M$, Finch L. Description and application of a system for locomotor rehabilitation. Med Biol Eng Comput. 1987;25:341-4.

3. Tyson SF, Rogerson L. Assistive walking devices in nonambulant patients undergoing rehabilitation after stroke: the effects on functional mobility, walking impairments, and patients' opinion. Arch Phys Med Rehabil. 2009; 90:475-9.

4. Pohl M, Mehrholz J, Ritschel C, Rückriem S. Speed-dependent treadmill training in ambulatory hemiparetic stroke patients: a randomized controlled trial. Stroke. 2002;33:553-8.

5. Scherer M. Gait rehabilitation with body weight-supported treadmill training for a blast injury survivor with traumatic brain injury. Brain Inj. 2007;21:93-100

6. Yen S-C, Schmit BD, Landry JM, Roth H, Wu M. Locomotor adaptation to resistance during treadmill training transfers to overground walking in human SCI. Exp Brain Res. 2012;216:473-82.

7. Mirelman A, Maidan I, Herman T, Deutsch JE, Giladi N, Hausdorff JM. Virtua reality for gait training: can it induce motor learning to enhance complex walking and reduce fall risk in patients with Parkinson's disease? The Journals of Gerontology: Series A. 2011;66:234-40.

8. Kim J, Park H-S, Damiano DL. An interactive treadmill under a novel control scheme for simulating overground walking by reducing anomalous force. IEEE/ASME Transactions on mechatronics. 2015;20:1491-6.

9. Kim J, Stanley CJ, Curatalo LA, Park H-S: A user-driven treadmill contro scheme for simulating overground locomotion. In Engineering in Medicine and Biology Society (EMBC), 2012 Annual international conference of the IEEE. IEEE; 2012: 3061-3064

10. Riley PO, Paolini G, Della Croce U, Paylo KW, Kerrigan DC. A kinematic and kinetic comparison of overground and treadmill walking in healthy subjects. Gait and posture. 2007:26:17-24.

11. Lee SJ, Hidler J: Biomechanics of overground vs. treadmill walking in healthy individuals. J Appl Physiol 2008, 104:747-755.

12. Magill RA, Anderson DI: Motor learning and control: concepts and applications. McGraw-Hill New York; 2007

13. Canning CG, Ada L, Paul SS. Is automaticity of walking regained after stroke? Disabil Rehabil. 2006;28:97-102.

14. Malone LA, Bastian AJ. Thinking about walking: effects of conscious correction versus distraction on locomotor adaptation. J Neurophysiol. 2010; 103:1954-62.

15. Wulf $\mathrm{G}$, Prinz W. Directing attention to movement effects enhances learning: a review. Psychon Bull Rev. 2001:8:648-60.

16. Wulf G, McNevin N, Shea $\mathrm{CH}$ : The automaticity of complex motor skill learning as a function of attentional focus. The Quarterly Journal of Experimental Psychology: Section A 2001, 54:1143-1154.

17. Hejrati B, Crandall KL, Hollerbach JM, Abbott JJ: Kinesthetic force feedback and belt control for the treadport locomotion interface. IEEE transactions on haptics 2015, 8:176-187.

18. Minetti AE, Boldrini L, Brusamolin L, Zamparo P, McKee T. A feedbackcontrolled treadmill (treadmill-on-demand) and the spontaneous speed of walking and running in humans. J Appl Physiol. 2003;95:838-43.

19. Christensen RR, Hollerbach JM, XU Y, Meek SG. Inertial-force feedback for the treadport locomotion interface. Presence: Teleoperators and Virtual Environments. 2000;9:1-14.

20. Feasel J, Whitton MC, Kassler L, Brooks FP, Lewek MD: The integrated virtual environment rehabilitation treadmill system. IEEE Transactions on Neural Systems and Rehabilitation Engineering 2011, 19:290-297.

21. Kim J, Gravunder A, Park H-S. Commercial motion sensor based low-cost and convenient interactive treadmill. Sensors. 2015;15:23667-83.

22. Bulea TC, Kim J, Damiano DL, Stanley CJ, Park H-S. Prefrontal, posterior parietal and sensorimotor network activity underlying speed control during walking. Front Hum Neurosci. 2015:9:247.

23. Arenth PM, Ricker JH, Schultheis MT. Applications of functional near-infrared spectroscopy (fNIRS) to neurorehabilitation of cognitive disabilities. Clin Neuropsychol. 2007;21:38-57.
24. Hamacher D, Herold F, Wiegel P, Hamacher D, Schega L. Brain activity during walking: a systematic review. Neurosci Biobehav Rev. 2015:57:310-27.

25. Gramigna V, Pellegrino G, Cerasa A, Cutini S, Vasta R, Olivadese G, Martino I, Quattrone A. Near-infrared spectroscopy in gait disorders: is it time to begin? Neurorehabil Neural Repair. 2017;31:402-12.

26. Herold F, Wiegel P, Scholkmann F, Thiers A, Hamacher D, Schega L. Functional near-infrared spectroscopy in movement science: a systematic review on cortical activity in postural and walking tasks. Neurophotonics. 2017:4:041403.

27. Leff DR, Orihuela-Espina F, Elwell CE, Athanasiou T, Delpy DT, Darzi AW, Yang G-Z. Assessment of the cerebral cortex during motor task behaviours in adults: a systematic review of functional near infrared spectroscopy (fNIRS) studies. Neuroimage. 2011:54:2922-36.

28. Khan RA, Naseer N, Qureshi NK, Noori FM, Nazeer H, Khan MU. fNIRS-based Neurorobotic Interface for gait rehabilitation. J Neuroeng Rehabil. 2018:15:7.

29. Huppert T, Schmidt B, Beluk N, Furman J, Sparto P. Measurement of brain activation during an upright stepping reaction task using functional nearinfrared spectroscopy. Hum Brain Mapp. 2013;34:2817-28.

30. Karim H, Schmidt B, Dart D, Beluk N, Huppert T. Functional near-infrared spectroscopy (fNIRS) of brain function during active balancing using a video game system. Gait and posture. 2012;35:367-72

31. Rosso AL, Cenciarini M, Sparto PJ, Loughlin PJ, Furman JM, Huppert TJ. Neuroimaging of an attention demanding dual-task during dynamic postural control. Gait and posture. 2017;57:193-8.

32. Vitorio $R$, Stuart $S$, Rochester $L$, Alcock $L$. Pantall a: fnirs response during walking - artefact or cortical activity? Neuroscience and Biobehavioral Reviews: A systematic review; 2017

33. Miyai I, Tanabe HC, Sase I, Eda H, Oda I, Konishi I, Tsunazawa Y, Suzuki T, Yanagida T, Kubota K. Cortical mapping of gait in humans: a near-infrared spectroscopic topography study. Neuroimage. 2001;14:1186-92.

34. Kim HY, Yang SP, Park GL, Kim EJ, You JSH. Best facilitated cortical activation during different stepping, treadmill, and robot-assisted walking training paradigms and speeds: a functional near-infrared spectroscopy neuroimaging study. NeuroRehabilitation. 2016:38:171-8.

35. Suzuki M, Miyai I, Ono T, Oda I, Konishi I, Kochiyama T, Kubota K. Prefrontal and premotor cortices are involved in adapting walking and running speed on the treadmill: an optical imaging study. Neuroimage. 2004;23:1020-6.

36. Mirelman A, Maidan I, Bernad-Elazari H, Nieuwhof F, Reelick M, Giladi N, Hausdorff JM. Increased frontal brain activation during walking while dual tasking: an fNIRS study in healthy young adults. J Neuroeng Rehabil. 2014;11:85.

37. Lu C-F, Liu Y-C, Yang Y-R, Wu Y-T, Wang R-Y. Maintaining gait performance by cortical activation during dual-task interference: a functional nearinfrared spectroscopy study. PloS one. 2015;10:e0129390.

38. Klempiŕ O, Krupička R, Mehnert J, Čejka V, Peterová K, Plaňanská E, Brožová H, Růžička E, Szabó Z, Jech R. P 024-near-infrared spectroscopy patterns of cortical activity during gait in Parkinson's disease patients treated with DBS STN. Gait and posture. 2018;65:273-5.

39. Holper L, Biallas M, Wolf M. Task complexity relates to activation of cortical motor areas during uni-and bimanual performance: a functional NIRS study. Neuroimage. 2009:46:1105-13.

40. Tanaka H, Katura T, Sato H. Task-related oxygenation and cerebral blood volume changes estimated from NIRS signals in motor and cognitive tasks. Neuroimage. 2014;94:107-19.

41. Mandrick K, Derosiere G, Dray G, Coulon D, Micallef J-P, Perrey S. Prefrontal cortex activity during motor tasks with additional mental load requiring attentional demand: a near-infrared spectroscopy study. Neurosci Res. 2013; 76:156-62.

42. Han C-H, Hwang H-J, Lim J-H, Im C-H. Assessment of user voluntary engagement during neurorehabilitation using functional near-infrared spectroscopy: a preliminary study. J Neuroeng Rehabil. 2018;15:27.

43. Harada T, Miyai I, Suzuki M, Kubota K. Gait capacity affects cortical activation patterns related to speed control in the elderly. Exp Brain Res. 2009;193: 445-54

44. Scholkmann F, Kleiser S, Metz AJ, Zimmermann R, Pavia JM, Wolf U, Wolf M. A review on continuous wave functional near-infrared spectroscopy and imaging instrumentation and methodology. Neuroimage. 2014;85:6-27.

45. Jasper $\mathrm{HH}$. The ten-twenty electrode system of the international federation. Electroencephalogr Clin Neurophysiol. 1958;10:370-5.

46. Ye JC, Tak S, Jang KE, Jung J, Jang J. NIRS-SPM: statistical parametric mapping for near-infrared spectroscopy. Neuroimage. 2009;44:428-47. 
47. Yogev-Seligmann G, Hausdorff JM, Giladi N. The role of executive function and attention in gait. Movement disorders: official journal of the Movement Disorder Society. 2008;23:329-42.

48. Kurz MJ, Wilson TW, Arpin DJ. Stride-time variability and sensorimotor cortical activation during walking. Neuroimage. 2012;59:1602-7.

49. Strangman G, Franceschini MA, Boas DA. Factors affecting the accuracy of near-infrared spectroscopy concentration calculations for focal changes in oxygenation parameters. Neuroimage. 2003;18:865-79.

50. Tak S, Yoon SJ, Jang J, Yoo K, Jeong Y, Ye JC. Quantitative analysis of hemodynamic and metabolic changes in subcortical vascular dementia using simultaneous near-infrared spectroscopy and fMRI measurements. Neuroimage. 2011;55:176-84.

51. Cohen J: Statistical power analysis for the behavioural sciences. Hillsdale, NJ erlbaum; 1988.

52. Hill A, Bohil C, Lewis J, Neider M: Prefrontal cortex activity during walking while multitasking: An fNIR study. In Proceedings of the Human Factors and Ergonomics Society Annual Meeting. SAGE Publications Sage CA: Los Angeles, CA; 2013: 1224-1228.

53. Holtzer R, Mahoney JR, Izzetoglu M, Izzetoglu K, Onaral B, Verghese J. fNIRS study of walking and walking while talking in young and old individuals. Journals of Gerontology Series A: Biomedical Sciences and Medical Sciences. 2011:66:879-87.

54. Gatouillat A, Bleton $H$, VanSwearingen J, Perera S, Thompson S, Smith T, Sejdić E. Cognitive tasks during walking affect cerebral blood flow signal features in middle cerebral arteries and their correlation to gait characteristics. Behav Brain Funct. 2015;11:29.

55. Jor'dan AJ, Poole VN, lloputaife I, Milberg W, Manor B, Esterman M, Lipsitz LA. Executive network activation is linked to walking speed in older adults: functional MRI and TCD ultrasound evidence from the MOBILIZE Boston study. Journals of Gerontology Series A: Biomedical Sciences and Medical Sciences. 2017;72:1669-75.

56. Clark DJ, Christou EA, Ring SA, Williamson JB, Doty L. Enhanced somatosensory feedback reduces prefrontal cortical activity during walking in older adults. Journals of Gerontology Series A: Biomedical Sciences and Medical Sciences. 2014:69:1422-8.

57. Koenraadt KL, Roelofsen EG, Duysens J, Keijsers NL. Cortical control of normal gait and precision stepping: an fNIRS study. Neuroimage. 2014;85: 415-22.

58. Song M, Kim J. An ambulatory gait monitoring system with activity classification and gait parameter calculation based on a single foot inertial sensor. IEEE Trans Biomed Eng. 2018;65:885-93.

Ready to submit your research? Choose BMC and benefit from:

- fast, convenient online submission

- thorough peer review by experienced researchers in your field

- rapid publication on acceptance

- support for research data, including large and complex data types

- gold Open Access which fosters wider collaboration and increased citations

- maximum visibility for your research: over $100 \mathrm{M}$ website views per year

At BMC, research is always in progress.

Learn more biomedcentral.com/submissions 